\title{
Antibody-Drug Conjugates
}

\author{
Ashutosh A. Kulkarni ' • Hovhannes J. Gukasyan ${ }^{2}$
}

Received: 5 August 2015 / Accepted: 10 August 2015 / Published online: 15 August 2015

(C) Springer Science+Business Media New York 2015

Antibody-drug conjugates (ADCs) are an exciting and new class of therapeutic modalities that have gained significant traction over the past few years - specifically in the field of oncology. With the recent approvals of CD-30 directed Adcetris ${ }^{\circledR}$ (brentuximab vedotin) and the Her2 targeted Kadcyla ${ }^{\circledR}$ (ado-trastuzumab emtansine) and hundreds of other ADCs in various stages of drug discovery and development, the field is experiencing significant optimism and interest from the biopharmaceutical industry. Several esteemed organizations including Celgene, Immunogen, Pfizer, Agensys, and Genentech - to name a few - are investing significant resources into ADC discovery and development. The lure of ADCs is that they combine the specificity of a monoclonal antibody with the potent cytotoxicity of a small molecule toxin payload. The hypothesis is that by combining these 2 characteristics, one should be able to specifically target the ADC to the diseased tissue and steer clear of the non-specific toxicity of the toxin payload. Also, the presence of the toxin payload would make the ADC significantly more efficacious compared to antibody alone. One of the key benefits is that the antibody does not necessarily need to have therapeutic activity on its own since the efficacy of $\mathrm{ADC}$ is driven by internalization of the ADC molecule $\rightarrow$ trafficking to the lysosome $\rightarrow$ degradation to the final catabolite $\rightarrow$ cytotoxic activity of the catabolite on the cellular machinery.

However, ADCs require multi-parameter optimization, which is one of the key challenges in their discovery and development effort. In fact, significant investment is placed during ADC discovery in identifying a viable target. The target is studied for its cellular trafficking, internalization characteristics (a necessity for $\mathrm{ADCs}$ ), and its expression profile to ensure

Ashutosh A. Kulkarni akulkarni@celgene.com

Celgene Corporation, San Diego, CA, USA

Pfizer Inc, San Diego, CA, USA that it is unique to the target tissue and has little to no expression in normal tissues. Dr Ben Xu, Dr Kate Lai, and Dr Puja Sapra have contributed excellent articles to this theme issue that focus on these very topics. Another aspect of the multiparametric optimization is the selection of appropriate linker and toxin payload combination. Selection of the appropriate linker and toxin payload is critical to achieving the required efficacy without giving rise to significant systemic toxicity. Important questions include: 1) which type of linker is suitable for the target of choice - cleavable or non-cleavable? 2) what is the impact of attaching a linker and payload to the conjugation site on the antibody? 3) which conjugation site on the antibody is ideal for covalent attachment of linker and payload? Dr Bruce Tomczuk has contributed a very informative article on the linker-payload chemistry aspects. In addition, Dr Roger Pak has also contributed a revealing article on the physicochemical considerations that have an impact on the formulation design and technology of ADCs.

Heterogeneity has been one of the Achilles heel in ADC drug discovery and development. Not only does it lead to analytical challenges in terms of quantitative or qualitative biophysics and chemistry, but it also leads to scale up and production challenges in late development making it very costly and time consuming. This is partly offset, however, by the high potency and selectivity of ADCs which translates to relatively lower therapeutic doses and drug substance, drug product requirements. As a result, site specific and well controlled conjugation approaches have been developed to make the ADCs more homogenous with well characterized drug-antibody ratios (DAR). In addition, cell-free systems are being utilized for production of antibodies to eliminate post-translational modifications and confer more homogeneity, better stability, as well as better pharmacokinetic characteristics on the ultimate ADC molecule. Dr Gang Yin's article on this aspect is cutting edge and an excellent addition to this theme issue.

The optimized ADC candidates are evaluated in preclinical ADMET studies as well as efficacy models. The ADMET 
studies for ADCs are generally more complex than those of small molecules or biologics, mainly because they combine both aspects into one. The analytical assays needed for ADC studies span the whole gamut of ligand binding assays (ELISA, Meso Scale) all the way to protein mass spectrometry. Logistics of sample processing and analysis are more involved and require multiple, special considerations. The ADME properties are also a hybrid of disposition characteristics of small molecules and those of biologics, making it very challenging to predictably translate data from preclinical species to man. Significant advances are being made in bioanalytical aspects as well as PKPD modeling and Dr Dowdy Jackson, Dr Dhaval Shah, and Dr Amrita Kamath have contributed key articles on these topics. These articles are a great resource for the readers of this issue.

Dr Barbara Mounho-Zamora, in her article, touches on the regulatory and nonclinical aspects of ADC development, and Dr Shen evaluates the role of ADCs in a non-oncology setting (ophthalmology). Even though there is scarce literature on the use of ADCs in non-oncology indications, there have been discussions on potential uses in inflammatory conditions. More literature on this will probably appear in the not-toodistant future.

Significant hurdles must be overcome in order to successfully prosecute an ADC program. Even though two ADCs have already been approved by the US FDA, the sheer cost of ADC discovery and development combined with the complexities and uncertainties that arise from relatively poor bench-tobeside predictability has competing organizations always thinking about the best utility of their resources. As long as early proof-of-concept can be achieved relatively rapidly in a Phase I study, with the appropriate biomarker and patient selection, organizations are willing to invest in these novel therapeutic modalities. With the current emphasis on cost effectiveness, organizations need confidence that their investment will be valuable, while patients and payers would be willing to pay for these state of the art, novel, and high value treatments. Several scientific meetings now have ADC focused sessions that are very well attended suggesting that the interest in this field is growing tremendously and healthcare providers from all aspects of the profession see value in it. World ADC summit organized by Hanson Wade is one such multi-day summit that is dedicated completely to ADCs. Additionally, the prospect of other, non-oncology related therapeutic applications of ADCs, when fully realized will unlock the full potential of this medical technology. As we exchange more ideas and our collective knowledge of the various ADC-related parameters increase, we will probably see a significant expansion of ADC therapies in non-oncology indications as well as several product approvals across various therapeutic indications for years to come. 